\title{
Capacitive Sensing of Local Bond Layer Thickness and Coverage in Thermal Interface Materials ${ }^{1}$
}

\author{
Stephen H. Taylor and Suresh V. Garimella ${ }^{2}$ \\ Cooling Technologies Research Center, an NSF I/UCRC \\ School of Mechanical Engineering \\ 585 Purdue Mall, Purdue University \\ West Lafayette, IN 47907 USA
}

\begin{abstract}
An instrumentation technique is developed using embedded capacitive sensors to measure the thickness and evenness of coverage of a thin layer of dielectric thermal interface material (TIM) between two substrates. The technique requires an array of sensors embedded into one substrate, with an electrically conductive opposing substrate. Local capacitance measurements are sensitive to both local bond layer thickness and local voiding. We propose a means for using an array of capacitance measurements to interpret both bond layer thickness and local voiding at every sensor location. An algorithm is developed which reveals both characteristics from a single set of capacitance measurements. Experiments are conducted with thermal grease layers of different bond layer thicknesses and void distributions using a prototype system constructed on printed circuit boards. The thickness and void distribution are successfully mapped across the bond layer using the algorithm developed. The technique offers a sensing approach for in situ instrumentation of layers of thermal grease in a thermal test vehicle.
\end{abstract}

Keywords: thermal interface material, TIM, TIM reliability, bond line thickness, thermal grease, embedded sensors, capacitance sensors

1. For International Journal of Heat and Mass Transfer (IJHMT)

2. Corresponding author, sureshg@purdue.edu

(C) 2016. This manuscript version is made available under the Elsevier user license http://www.elsevier.com/open-access/userlicense/1.0/ 


\section{Introduction}

\subsection{Background}

Thermal interface materials (TIMs) are an essential component in modern electronics packaging architectures. Thermal management of heat-producing electronic devices requires efficient heat transport through solid-solid interfaces. This typically necessitates placement of a high-conductivity, compliant medium at the interface to compensate for the deleterious effects of surface roughness and non-planarity of the mating substrates [1]. Significant commercial effort has been targeted at the development of new TIMs for improving bulk conductivity, wettability/adhesion, and long-term reliability. A TIM bond layer exhibiting poor bond-layer coverage may lead to failure of the electronics due to overheating. In their recent review of TIM reliability, Due and Robinson [2] observed that critical long-term reliability performance data on TIMs is not generally available. In the research community, it has proven difficult to gather end-of-life performance results in a consistent manner from diverse research groups [3]. Predicting TIM performance over the application lifetime based on projections from accelerated fatigue tests is also challenging $[3,4]$. Many techniques, such as C-Mode Scanning Acoustic Microscopy (CSAM) [5, 6], radiography [7], infrared microscopy [7], scanning electron microscopy [8], and transient thermal tomography [9], have been employed in an effort to characterize the physical integrity of TIM bond layers while under stress, or after being stressed.

In industry, practical considerations often preclude physical defects from being characterized directly; instead, thermal performance is characterized as a proxy. The bulk conductivity of TIMs may be ascertained through an ASTM D5470 standard test [10]. However, thermal test vehicles (TTVs) are critical for long-term reliability characterization for electronics applications. A TTV may be constructed to mimic critical aspects of the intended service condition $[2,3]$ and generally includes confining substrates, a heating element, a TIM layer, and temperature measurement instrumentation. Power cycling may be conducted within a TTV and approximate values for overall thermal resistance obtained over the course of the test. While such thermal test vehicles may provide a means for accurately producing the desired thermomechanical stresses, it is very difficult to monitor the evolution of bond layer thickness and 
bond layer coverage during the tests. Bond layer thickness may change globally over the footprint of the TIM, and/or suffer from more localized variations, as thermomechanical strain influences the mating substrates characterized by differing coefficients of thermal expansion (CTE). Bond layer coverage degrades as the TIM loses contact with the mating substrates during power cycling. In TIMs made from greases, thermomechanical action may cause material to exit the joint, resulting in voiding near the perimeter of the bond layer, in a process that is referred to as pump-out. In a standard TTV, which lacks means for in situ visualization of these phenomena, physical changes in the TIM cannot be characterized or interpreted in tandem with the thermal performance.

\subsection{Capacitive sensor concept}

Impedance sensors have been used in a wide variety of applications for observing liquid-gas phase fractions in microscale flows [11, 12], free surfaces [13], and flows between parallel surfaces [14]. In the last of these examples, an orthogonal mesh consisting of embedded rakes of electrodes on both substrates was used in order to produce a grid of crossing points across the mating area. The efficacy of such an approach has been demonstrated for the detection of dielectric anomalies in a $25 \mu \mathrm{m}$ interfacial gap [15]. While the orthogonal mesh concept cannot be readily implemented in a TTV, an alternative concept is proposed which is more conducive to this implementation. As illustrated in Figure 1, a bed of capacitive sensors is manufactured onto one of the substrates. A matrix of electrical pads resides on the outer surface of the die in a TTV, and is connected to external controls which allow for independent actuation of each pad with a sender signal. The (typically electrically conductive) heat sink or heat spreader mounted on top of the die is used to capture the receiving signal. Thus, the capacitance across the bond layer between any given sensor pad and the opposing wall can be measured. The array of capacitance sensors may be used to monitor the bond layer with good spatial resolution during the course of long-term reliability testing. 


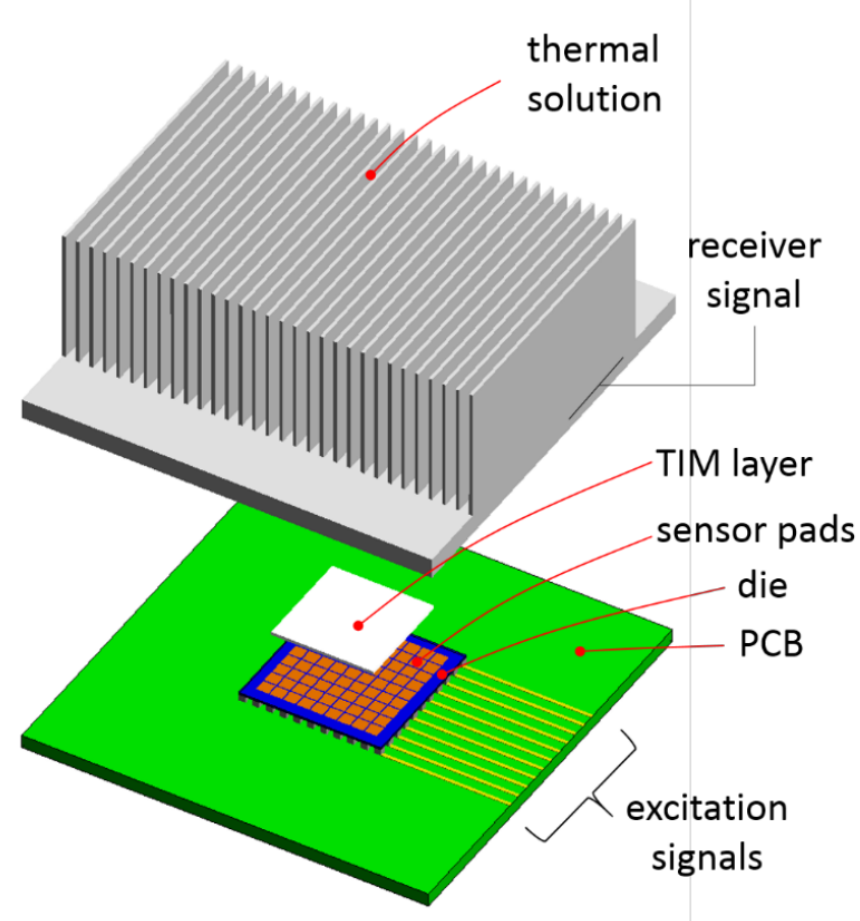

Figure 1. Conceptual illustration of thermal test vehicle with embedded capacitive sensors.

Capacitance measurements in this arrangement are sensitive to both the bond layer thickness $H$, and voiding within the TIM layer. Capacitance will decrease with either increasing $H$ or increasing void content. The combined effects of the two physical phenomena render it impossible to separately establish the thickness or the extent of voiding from a single capacitance measurement. However, we propose that when an array of $M$ capacitance measurements is obtained and analyzed collectively, information regarding bond layer size and distribution of void content may be accurately distinguished. In order to achieve this, an appropriate distinguishing algorithm must be developed to reconstruct maps of both characteristics using the single set of measurements, as outlined in Figure 2. 


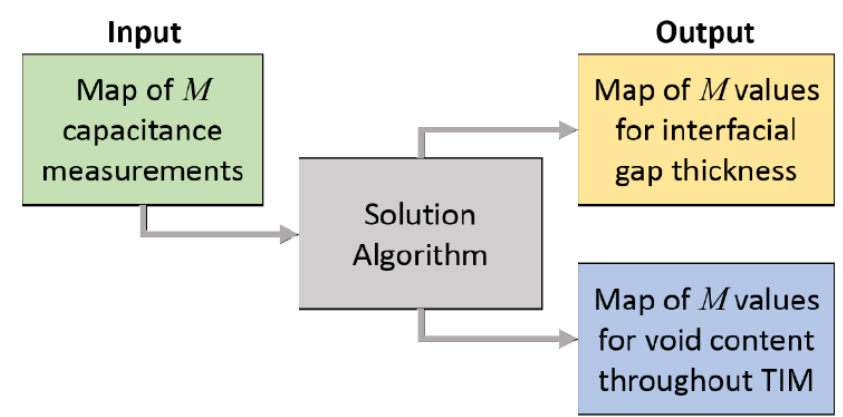

Figure 2. Illustration of solution algorithm objective.

In this work, a prototype system manufactured on printed circuit board (PCB) substrates is used for demonstration. An algorithm that distinguishes between voiding and thickness in bond layer variations proposed and its use demonstrated with experimental data. Measurements of both bond layer thickness and TIM coverage are demonstrated using the capacitive concept. Finally, the algorithm is used to experimentally acquire maps of evolving bond layer thickness and TIM coverage over the course of mechanical loading in a voided grease layer.

\section{Experimental Methods}

The prototype experimental system is manufactured on PCB substrates. Copper electrode pads are fabricated on the bottom substrate, which is shown in Figure 3a. The bottom substrate is a large 6layer board; multiplexer contacts are exposed on the underside and the 9 by 9 array of $1.5 \mathrm{~mm}$ by $1.5 \mathrm{~mm}$ copper pads are exposed on the upper side (Figure 3b). Electrode pads around the border of the array are permanently grounded, such that only the internal 7 by 7 array of 49 pads serve as active electrodes. A standard dielectric coating material applied to the entire surface fills the spaces between coplanar electrodes, and is then ground down to expose the electrode pads as flush-mounted surfaces. Nylon alignment pins mounted normal to the surface mate with holes in the top substrate. The top substrate is a second printed circuit board containing a single large electrode pad (Figure 3c), which mounts to the bottom substrate using spring-loaded screws fastened directly into tapped holes in the bottom substrate. 
Shim spacers ranging in thickness from $51 \mu \mathrm{m}$ to $191 \mu \mathrm{m}$ are introduced between the substrates to obtain known bond layer thicknesses for calibration.

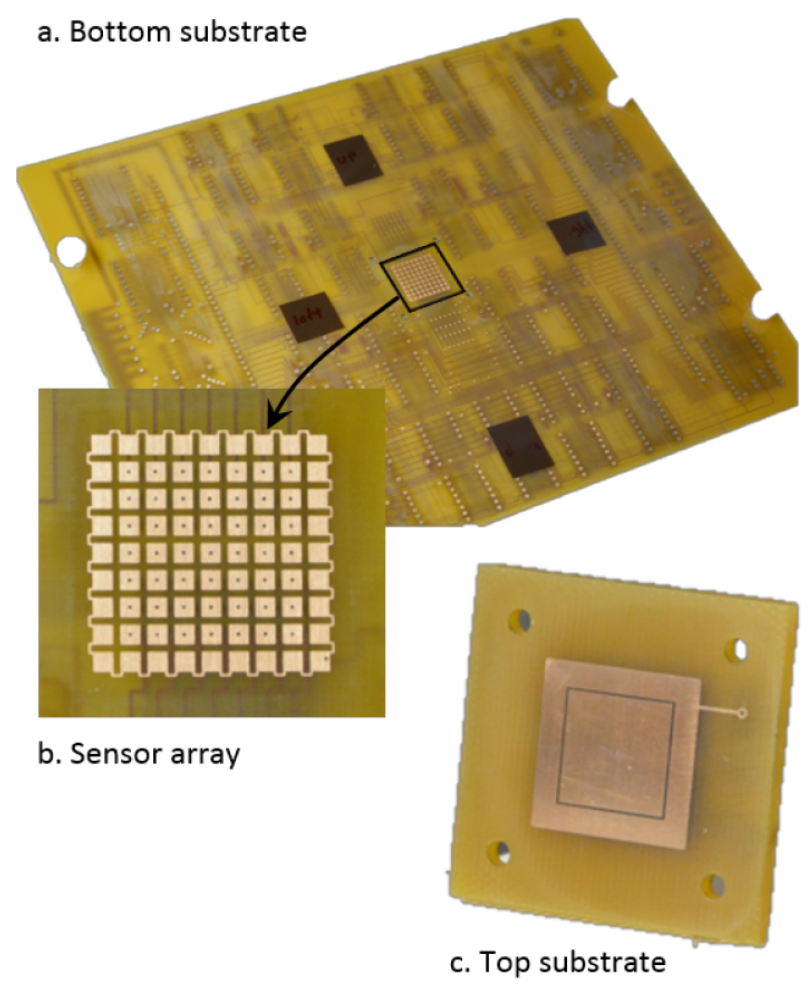

Figure 3. Prototype embedded sensor system manufactured on printed circuit boards.

Capacitance is measured using the charge transfer method, where the sender electrode is driven with an excitation signal and inductive current is measured at the receiving electrode. Other electrodes are held at virtual ground. A commercially available chip (AD7746) is used to perform the measurements at an excitation frequency of $32 \mathrm{kHz}$ [16]. The excitation signal is demultiplexed with standard analog integrated circuits (soldered onto the underside of the PCB) to allow for 6-switch digital control over the route of the signal. The top electrode serves as the receiver electrode. Capacitance is obtained as the mean of approximately 15 readings for each measurement. Measurement noise is approximately $\pm 2 \mathrm{fF}$. The thermal grease used for testing is Tgrease 2500, with a reported dielectric constant of $\varepsilon_{g}=5.7$ [17]. 


\section{Modeling Approach}

\subsection{System behavior model}

When implemented in a thermal test vehicle, the electrode size $s$ will likely be comparatively larger than bond layer thickness, $H$. In the prototype system, $s=1500 \mu \mathrm{m}$. Because of this large ratio, TIM coverage over individual electrodes may be approximated as two-dimensional. Therefore, capacitance at that electrode is a linear sum of capacitance across the TIM region and the voided region. Thus, at a given electrode $m$, with bond layer thickness $H_{m}$, the capacitance is expected to be proportional to the local void fraction $\chi_{m}$. Another result of the large dimensional ratio of the system is that electrode pads behave similarly to a parallel-plate capacitor. Specifically, the capacitance between a given electrode pad and the opposing substrate is inversely proportional to the separation distance, or bond layer thickness $H$. In light of these considerations, the capacitance obtained from an given electrode pad $m$ may be modeled using the relationship,

$$
C_{m}=\chi_{m}\left(\frac{J_{1, m}}{H_{m}}+J_{2, m}\right)+\left(1-\chi_{m}\right)\left(\frac{K_{1, m}}{H_{m}}+K_{2, m}\right) .
$$

For a parallel-plate model, $J_{1, m}=\varepsilon_{o} A_{m}, K_{1, m}=\varepsilon_{g} A_{m}$, and $J_{2, m}=K_{2, m}=0$, where $A_{m}$ is the area of the electrode pad. Accuracy is improved when values for $J_{1}, J_{2}, K_{1}$, and $K_{2}$ are found through a calibration process (discussed below in Section 4). The analysis here is carried out with normalized parameters, using a fixed reference value of $H_{\text {ref }}$ and two different void fractions of 0 and 1 , to provide respective reference capacitance measurements of $C_{m, g}=\left.C_{m}\right|_{H=H \mathrm{ref}, \chi=0}$ and $C_{m, o}=\left.C_{m}\right|_{H=H \mathrm{ref}, \chi=1}$. Normalized capacitance measurements may then be expressed as

$$
C_{m}^{*}=\frac{C_{m}-C_{m, o}}{C_{m, g}-C_{m, o}}=\frac{\eta^{-1}\left(\kappa_{m, 1}\left(1-\chi_{m}\right)+\chi_{m}\right)+\kappa_{m, 3}\left(\kappa_{m, 2}-1\right)\left(1-\chi_{m}\right)-1}{\kappa_{m, 1}+\kappa_{m, 3}\left(\kappa_{m, 2}-1\right)-1},
$$

where $\eta=H / H_{r e f}, \kappa_{1}=K_{1} / J_{1}, \kappa_{2}=K_{2} / J_{2}$, and $\kappa_{3}=J_{2} H_{r e f} / J_{1}$.

The solution algorithm should solve for the values of $\eta$ and $\chi$ in order to satisfy Equation 2.

Because $2 M$ variables are desired with only $M$ constraints, other expected characteristics of the system must be considered to arrive at an accurate solution. In the solution proposed in this work, the problem is 
conditioned by application of the following assumptions regarding characteristics of a normal grease bond layer:

1. A majority of sensors are not exposed to void content; therefore, a majority of values of $\chi$ are zero or very close to zero for any given measurement set.

2. Variations in $H$ result from substrate non-planarity and/or substrate warpage, which are always manifest as global effects with smooth, shallow gradients, and therefore may be fitted to a smooth function over the $x-y$ bond layer domain,

$$
\eta_{m}=f\left(x_{m}, y_{m}\right)
$$

3. Variations between $\chi$ values are generally manifest as local effects with no particular global gradient or pattern.

For this work, the function $f$ is chosen to be a five-coefficient quadratic function, defined by coefficient vector $\boldsymbol{a}$ as

$$
f_{a}(x, y)=a_{1}+a_{2} x+a_{3} y+a_{4} x^{2}+a_{5} x y+a_{6} y^{2}
$$

\subsection{Solution algorithm}

The algorithm proposed here consists of three sequential processes, as described in the following sections.

\section{Part 1: Iterative selection of voided locations}

Part 1 of the algorithm is a simple iterative fitting process. The initialization ( $\operatorname{step} p=0$ ) of the algorithm is to estimate the bond layer under the assumption of no void content $(\chi=0)$ for all sensor locations. This initial bond layer thickness map $\eta^{o}$ is solved directly as

$$
\eta_{m}^{o}=\frac{\kappa_{m, 1}}{C_{m}^{*}\left(\kappa_{m, 1}+\kappa_{m, 3}\left(\kappa_{m, 2}-1\right)\right)-\kappa_{m, 3}\left(\kappa_{m, 2}-1\right)+1}
$$

The coefficient vector $\boldsymbol{a}$ is then found using a least-squares method to best satisfy Equation 3. Any measurements affected by voiding will produce incorrectly high values of $\eta^{\circ}$ that contrast with 
neighboring values. Thus, for a value of $\eta^{o}$ above the curve-fit, void content is suspect. The measurement $c$ with the greatest contrast is identified,

$$
c=m_{\max }=\underset{\max }{\operatorname{Arg}}\left[\eta_{m}^{p}-f_{a}^{p}\left(x_{m}, y_{m}\right)\right]
$$

If the difference between the calculated value of $\eta_{m}$ and the curve-fit value is greater than the bond thickness uncertainty $u_{\eta}$,

$$
\eta_{c}^{p}-f_{a}^{p}\left(x_{c}, y_{c}\right)>u_{\eta}
$$

then measurement $c$ is categorized as a location with void content.

Iterations of the algorithm are carried out by using all locations currently considered non-voided to calculate $\eta$, while the function $f_{a}(x, y)$ is evaluated for locations that have been identified as containing void content:

$$
\eta_{m}^{p}=\left\{\begin{array}{cc}
f_{a}^{p-1}\left(x_{m}, y_{m}\right) & m \text { is voided } \\
\frac{\kappa_{m, 1}}{C_{m}^{*}\left(\kappa_{m, 1}+\kappa_{m, 3}\left(\kappa_{m, 2}-1\right)\right)-\kappa_{m, 3}\left(\kappa_{m, 2}-1\right)+1} & m \text { is not voided }
\end{array} .\right.
$$

The updated list of $\eta$ values are then used to fit the coefficient vector $\boldsymbol{a}$ and define $f_{a}{ }^{p}$. Although more than one location may satisfy Equation 7, it is found that best results are obtained when only one entry is labeled as containing void content per iteration, as some $\eta$ values may stand out above early curve-fits, but fall within the uncertainty of later curve-fits. The algorithm is complete when no more locations are labeled as containing void content.

\section{Part 2: Void content penalty function}

In Part 2 of the algorithm, the solution from Part 1 is used a starting point for an optimization routine. The routine solves for a vector containing the void fraction values $\chi$ and the curve-fit coefficients $\boldsymbol{a}$ as free variables, with a penalty function associated with total void content. By penalizing void content, this routine suppresses small values of $\chi$ and seeks to characterize the global pattern of capacitance measurements through the coefficients of the curve fit $f_{a}$. A simple quadratic penalty function is used in 
this work, which facilitates formulation of an analytical expression for the objective function gradient. The penalty function is

$$
P(\chi)=\left(P_{\chi}-\sum_{m}^{M} \chi_{m}\right)^{2} .
$$

The overall optimization objective $B$ may be written

$$
B(\chi, \boldsymbol{a})=P(\chi)+\sum_{m}^{M}\left(C_{i}^{*}-\frac{\left(\frac{\kappa_{m, 1}\left(1-\chi_{m}\right)+\chi_{m}}{f_{a}\left(x_{m}, y_{m}\right)}\right)+\kappa_{m, 3}\left(\kappa_{m, 2}-1\right)\left(1-\chi_{m}\right)-1}{\kappa_{m, 1}+\kappa_{m, 3}\left(\kappa_{m, 2}-1\right)-1}\right)^{2} .
$$

Expressions for $\partial B / \partial \chi_{m}$ and $\partial B / \partial a_{i}$ may be obtained and used to formulate $\nabla B$ for the optimization routine. A clipped Landweber method is suggested, where a variable step size $\alpha$ is used as the routine progresses,

$$
\left(\begin{array}{l}
\boldsymbol{\chi} \\
\boldsymbol{a}
\end{array}\right)^{p}=\left(\begin{array}{l}
\boldsymbol{\chi} \\
\boldsymbol{a}
\end{array}\right)^{p-1}-\alpha^{p} \nabla B
$$

Values for $\chi$ are kept within physical bounds by clipping at each step:

$$
\chi_{m} \leftarrow\left\{\begin{array}{lr}
1 & \chi_{m} \geq 1 \\
\chi_{m} & 0<\chi_{m}<1 \\
0 & 0 \leq 1
\end{array}\right.
$$

In this work, $P_{\chi}$ is found empirically for each individual test case as the value which results in the same total void content prediction as that previously given by Part 1 of the algorithm. The result is reallocation of void content away from locations where measurements may be matched instead with an improved curve-fit $f(x, y)$ toward locations with more significant values of void content.

\section{Part 3: Void content thresholding}

After application of the iterative fitting and conditioning the result with a penalty function, thresholding is applied. Thresholding is applied to the void fraction map at an uncertainty level for void fraction $u_{\chi}$ as 


$$
\chi_{m} \leftarrow\left\{\begin{array}{ll}
0 & \chi_{i}<u_{\chi} \\
\chi_{m} & \chi_{i} \geq u_{\chi}
\end{array} .\right.
$$

After thresholding, coefficients $\boldsymbol{a}$ are refitted using only electrodes for which $\chi=0$. All values of $\eta$ are then solved with the curve-fit $\eta_{m}=f_{a}\left(x_{m}, y_{m}\right)$, and then void fraction values which were above the threshold are solved using Equation 2. The thresholding process is iterated, and is observed in this work to converge to within a tolerance of 0.001 on $\chi$ values in merely 2-3 iterations. By thresholding, false positives for void content are eliminated, and slight improvements in the map of $\eta$ values are obtained.

\section{Experimental Demonstration}

The prototype system discussed in Section 1 is used to experimentally demonstrate the capability of the proposed system. Three calibration thicknesses are used, with $H=51,102$, and $191 \mu \mathrm{m}$. At each value of $H$, measurements are taken with all 49 electrodes for a completely voided condition $(\chi=1)$. The substrates are disassembled and the process is repeated three times to assess the repeatability of the measurements. A similar calibration process is used for the case with a non-voided grease bond layer $(\chi=$ 0 ). A shim template is used to manually dispense a layer of grease approximately $50 \%$ thicker than the intended bond layer onto the top substrate, which is then assembled onto the bottom substrate using a spacer to produce the correct bond layer thickness. Calibration curves are obtained using the data as

$$
\begin{aligned}
& C_{m, \chi=1} \cong\left(\frac{J_{1, m}}{H_{m}}+J_{2, m}\right) \quad \text { and } \\
& C_{m, \chi=0} \cong\left(\frac{K_{1, m}}{H_{m}}+K_{2 . m}\right)
\end{aligned}
$$

Figure 4 illustrates the calibration curves obtained for the first electrode in the array $(m=1)$ with curves referenced to $J_{2,1}$. It is noted that sensitivity to $H$ increases dramatically as $H \rightarrow 0$, with the practical consequence that theoretically achievable resolution in $H$ improves with respect to measurement noise. However, in the prototype system, the uncertainty due to repeatability associated with reassembly prevents accurate calibration for bond layers thinner than $50 \mu \mathrm{m}$. Repeatability in terms of bond layer 
thickness when using the shim spacers is approximately constant at $\pm 10 \mu \mathrm{m}$ at all three calibration points. When producing calibration curves, points at larger values of $H$ are weighted more heavily in order to account for the significant differences in uncertainty magnitude in terms of capacitance. The reference value used for bond layer thickness is $H_{r e f}=100 \mu \mathrm{m}$.

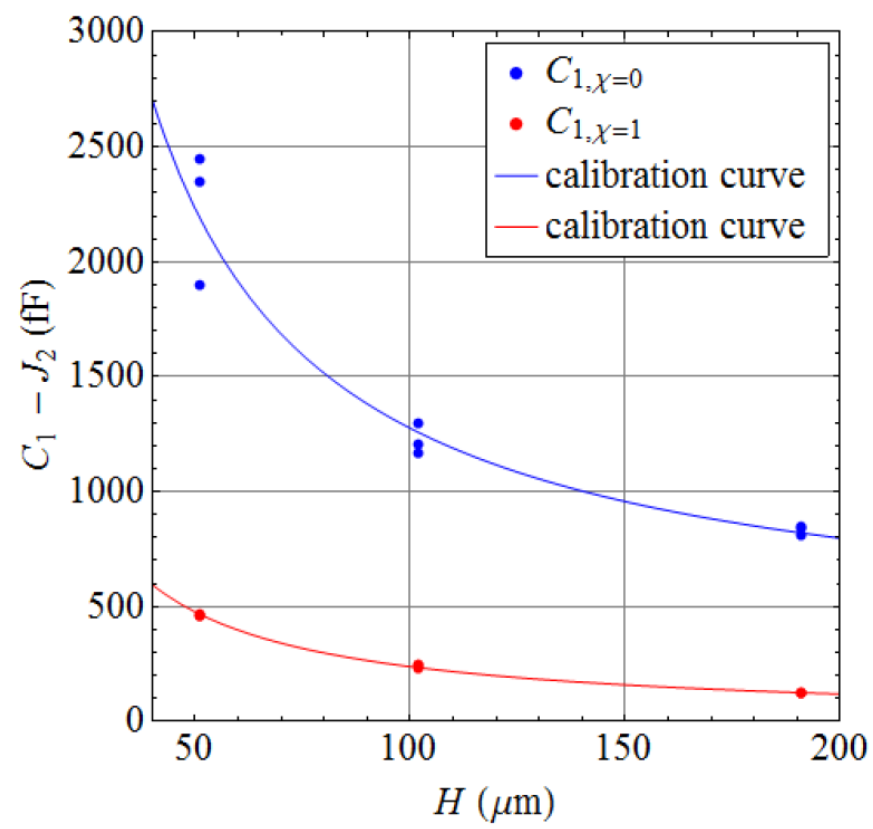

Figure 4. Example calibration curves for a single capacitance sensor, with $J_{1}=0.0238, K_{1}=0.0957, K_{2}-$ $J_{2}=319$.

Measurement of a changing, nonuniform bond layer thickness is demonstrated in Figure 5. Spatial locations across the bond layer are described using the cardinal directions illustrated by the compass icon in Figure 5a. In this test, a grease layer of $254 \mu \mathrm{m}$ is dispensed onto the top substrate and the substrates are assembled without a spacer. The spring-loaded screws at the four corners are tightened to induce increasingly asymmetric mechanical loads on the grease, changing the layer thickness. The solution algorithm is used to obtain maps of bond layer thickness $\eta$, which are shown with diagrams of the forces induced at the substrate corners. The force of each spring is calculated using the known compression and spring constant. 


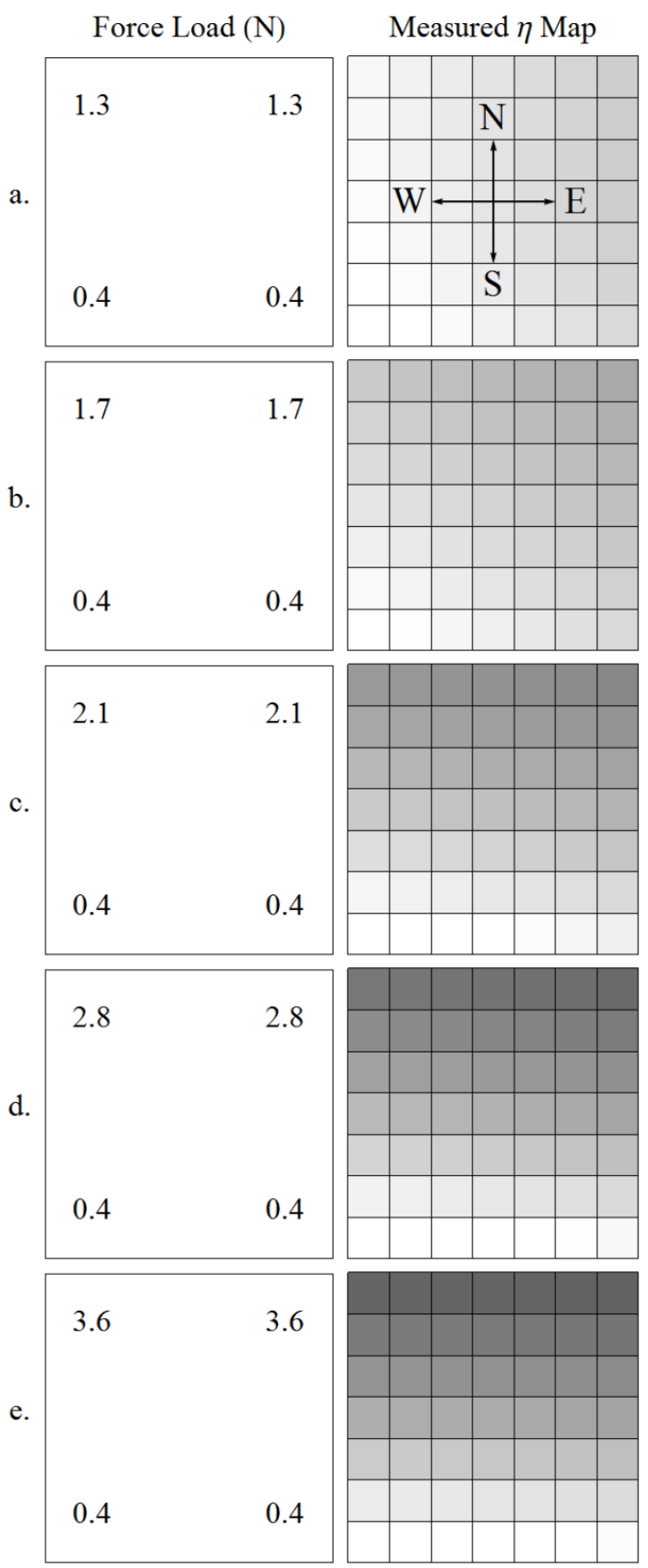

Figure 5. Experimental case with increasing load in the northwest and northeast locations. Grayscale range is from white at $\eta=2$ to black at $\eta=0$, with $H_{\text {ref }}=100 \mu \mathrm{m}$. 
Because the substrates are assembled without a spacer, the initial state (Figure 5a) of the substrate is slightly tilted. Thus, the bond layer begins with nonuniform thickness of $H=207 \mu \mathrm{m}$ at the southwest corner and $H=161 \mu \mathrm{m}$ at the northeast corner in Figure 5a. The bond layer proceeds to reduce in thickness at the north side, and increase at the southeast corner, as force is applied at the northern corners. The final measured bond layer thickness is $197-211 \mu \mathrm{m}$ along the south edge and 77-79 $\mu \mathrm{m}$ along the north edge (Figure 5e).
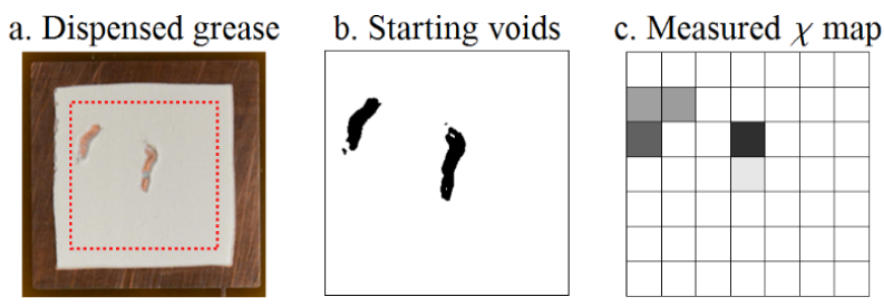

Figure 6. Experimental case with voided grease layer: (a) Optical image of original voids in dispensed grease (red outline indicates top electrode); (b) void locations identified from optical image; (c) void fraction map obtained from the solution algorithm after assembly. Grayscale range is from white at $\chi=0$ to black at $\chi=0.5$, with $H_{r e f}=100 \mu \mathrm{m}$.

Figure 6 illustrates the use of the capacitance sensors to image a TIM layer containing two voids. Voids are created by removing some grease after dispensing onto the top substrate (Figure 6a). From the optical image, an image of the grease voids is obtained by contrast thresholding (Figure 6b). When the substrates are assembled and pressure is applied, voids in the grease remain. The void fraction value map obtained from application of the algorithm to the experimental capacitance measurements is shown in Figure $6 \mathrm{c}$ for comparison. It is noted that the shape and size of the void structures is affected by the assembly process. However, it is clearly observed that the void structures are captured and distinguished by the measurements. This experiment was performed using a $76 \mu \mathrm{m}$ spacer. The solution algorithm identified the bond layer thickness of this case as 85-92 $\mu \mathrm{m}$ throughout the layer (not shown). The discrepancy is attributed primarily to the physical uncertainties in the prototype experimental system as opposed to inaccuracy of the algorithm. With this demonstration, both bond layer thickness and void content have been successfully characterized using the algorithm. It is noted that in previous work, 
grayscale void fraction maps such as Figure $6 \mathrm{c}$ have been used to generate a pseudo-high-resolution binary image of the estimated void shape, [18].

A demonstration of bond layer thickness and void content evolution is presented in Figure 7. In this test, a dispensed grease layer of $254 \mu \mathrm{m}$ is applied to the substrate and two voids are created, labeled Void A and Void B (Figure 7a). Increasing pressure is applied to the north side of the system, with measurements obtained after different applied pressure increments. The algorithm is used to reconstruct maps of bond layer thickness and void fractions for each measurement set. The first void fraction map (Figure $7 \mathrm{~b}$ ) illustrates the two voids clearly. In addition, this map includes a measurable void fraction value for a sensor on the north side, which may be due to a flaw in the dispensed layer or a void formed during assembly. As pressure is increased, grease flows toward the perimeter, eliminating Void B and the small void on the north side (Figure 7c). As pressure increases on the northern side of the system, flow is induced in the grease, carrying Void A along until it is near the south edge of the sensing area (Figure 7dg). The system delivers data in stages, including the thickness distribution of the bond layer and the spatial distribution of void regions. After the test, the substrates are separated to reveal Void A visibly observable at the south side of the bond area (Figure 7h), as indicated by the final measurement (Figure $7 \mathrm{~g})$.

The experimental results from the prototype system provide proof-of-concept confirmation that both bond layer thickness and bond line coverage of a dielectric TIM may be distinguished through an array of capacitance measurements. When the capacitive sensor system is implemented on a silicon wafer in practice, a denser array of smaller electrodes may be used. In conjunction with a high-precision calibration process, dramatic improvements in resolution are possible. 


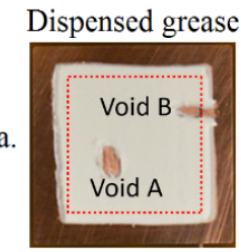

Force Load $(\mathrm{N})$

Measured $\eta$ Map Measured $\chi$ Map

b.

$0.9 \quad 0.9$

0.4

0.4

$1.3 \quad 1.3$

c.

$0.4 \quad 0.4$

$\begin{array}{ll}1.7 & 1.7\end{array}$

d.

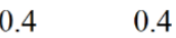

$2.1 \quad 2.1$

c.

$0.4 \quad 0.4$

2.8

2.8

f.

$0.4 \quad 0.4$

g.

\begin{tabular}{ll}
3.6 & 3.6 \\
0.4 & 0.4 \\
\hline
\end{tabular}

After test

h.

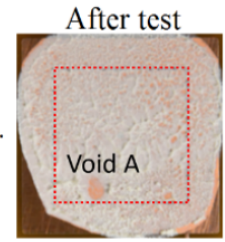

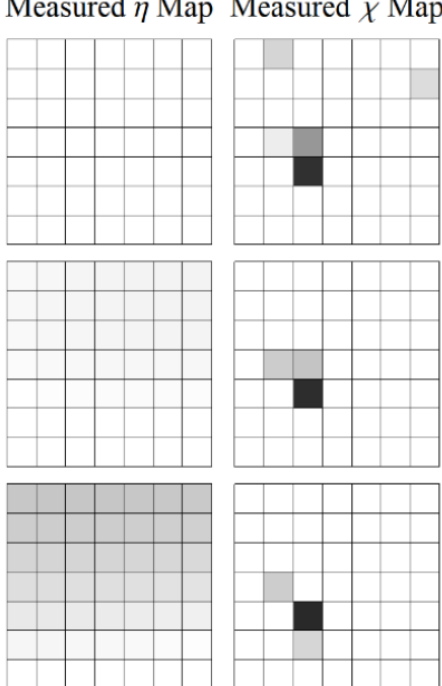
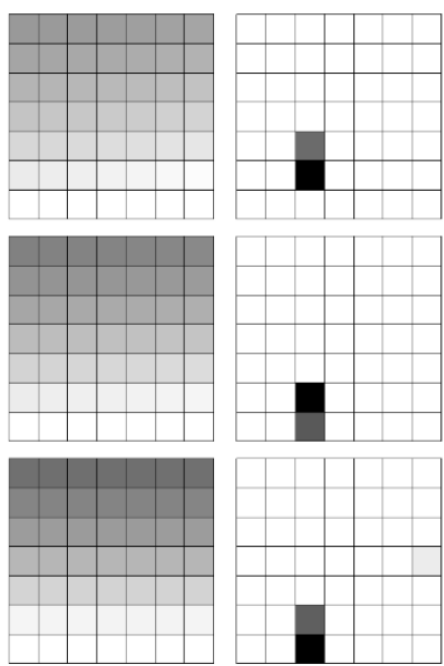

Figure 7. Experimental results for initially voided grease layer with increasingly non-uniform pressure (red outline indicates top electrode). Grayscale range is from white at $\eta=2$ to black at $\eta=0$, and white at $\chi=0$ to black at $\chi=0.5$, with $H_{r e f}=100 \mu \mathrm{m}$. 


\section{Conclusion}

The embedded capacitance sensing method demonstrated provides a viable means for characterizing a thin dielectric layer, such as a thermal grease, to detect the spatial distribution of thickness and voiding. The solution algorithm proposed successfully reproduces both bond layer thickness distribution and bond layer coverage from a single set of capacitance measurements. Because measurements may be obtained in situ, the technique is ideal for instrumentation of temporally evolving systems. If implemented in a thermal test vehicle, overall substrate warpage and loss of bond layer coverage due to pump-out would be obtained directly, adding valuable insight into the relationships between thermal resistance, thermomechanical stress, and physical bond layer integrity.

\section{Acknowledgements}

The authors gratefully recognize financial support for this work from Cooling Technologies Research Center, a National Science Foundation Industry/University Cooperative Research Center at Purdue University, as well as technical discussions with Dr. Justin Weibel. 
Capacitive Sensing of Local Bond Layer Thickness and Coverage in Thermal Interface Materials

\section{References}

[1] S. V. Garimella, A. S. Fleischer, J. Y. Murthy, A. Keshavarzi, R. Prasher, C. Patel, S. H. Bhavnani, R. Venkatasubramanian, R. Mahajan, Y. Joshi, B. Sammakia, B. A. Myers, L. Chorosinski, M. Baelmans, P. Sathyamurthy and P. E. Raad, "Thermal Challenges in Next-Generation Electronic Systems," IEEE Transactions on Components and Packaging Technologies, vol. 31, no. 4, pp. 801$815,2008$.

[2] J. Due and J. A. Robinson, "Reliability of Thermal Interface Materials: A Review," Applied Thermal Engineering, vol. 50, pp. 455-463, 2013.

[3] N. Goel, T. K. Anoop, A. Bhattacharya, J. A. Cervantes, R. K. Mongia, S. V. Machiroutu, L. HauLan, Y. C. Huang, K. C. Fan, B. L. Denq, C. H. Liu, C. H. Lin, C. W. Tien and J. H. Pan, "Technical Review of Characterization Methods for Thermal Interface Mateials," in 11th Intersociety Conference on Thermal and Thermomechanical Phenomena in Electronic Systems (ITHERM), Orlando, FL, USA, May 28-31, 2008.

[4] R. Prasher, "Thermal Interface Materials: Historical Perspective, Status, and Future Directions," Proceedings of the IEEE, vol. 94, no. 8, pp. 1571-1586, 2006.

[5] S. Haque, G. Q. Lu, J. Goings and J. Sigmund, "Characterization of Interfacial Thermal Resistance by Acoustic Micrography Imaging," Microelectronics Reliability, vol. 40, pp. 465-476, 2000.

[6] N. Islam, S. Lee, J. Lee, Y. Ka, J. Khim and J. Galloway, "TIM Selection Methodology for High Power Flip Chip Packages," in 12th Intersociety Conference on Thermal and Thermomechanical Phenomena in Electronic Systems (ITHERM), Las Vegas, NV, USA, June 2-5, 2010.

[7] A. Gupta, Y. Liu, N. Zamora and T. Paddock, "Thermal Imaging for Detecting Thermal Interface Issues in Assembly and Reliability Stressing," in 10th Intersociety Conference on Thermal and Thermomechanical Phenomena in Electronics Systems (ITHERM), San Diego, CA, USA, May 30 June 2, 2006.

[8] A. Gowda, D. Esler, S. Tonapi, A. Zhong, K. Srihari and F. Schattenmann, "Micron and SubmicronScale Characterization of Interfaces in Thermal Interface Material Systems," Journal of Electronic Packaging, vol. 128, pp. 130-136, 2006.

[9] H. Erturk, "Characterization of Electronic Packages by Thermal Diffusion Tomography," in ASME Heat Transfer Summer Conference, San Fransisco, CA, July 19-23, 2009.

[10] A. D5470-12, Standard Test Method for Thermal Transmission Properties of Thermally Conductive Electrical Insulation Materials, West Conshohocken, PA: ASTM International www.astm.org, 2012.

[11] P. Valiorgue, S. N. Ritchey, J. A. Weibel and S. V. Garimella, "Design of a Non-Intrusive Electrical Impedance-Based Void Fraction Sensor for Microchannel Two-Phase Flows," Measurement Science and Technology, vol. 25, no. 9, p. 095301, 2014.

[12] P. Gijsenbergh and R. Puers, "Permittivity-Based Void Fraction Sensing for Microfluidics," Sensors and Actuators A: Physical, vol. 195, pp. 64-70, 2013.

[13] S. Thiele, M. J. Da Silva and U. Hampel, "Capacitance Planar Array Sensor for Fast Multiphase Flow Imaging," IEEE Sensors Journal, vol. 9, no. 5, pp. 533-540, 2009.

[14] M. J. Da Silva and U. Hampel, "A Field-Focusing Imaging Sensor for Fast Visualization of Multiphase Flows," Measurement Science and Technology, vol. 20, 2009.

[15] S. H. Taylor and S. V. Garimella, "Void Detection in Dielectric Films using a Floating Network of Substrate-Embedded Electrodes," Journal of Electronic Packaging, vol. 136, no. 4, p. 041008, 2014.

[16] A. D. Inc., "24-Bit Capacitance-to-Digital Converter with Temperature Sensor: AD7745/7746," Data Sheet, no. Norwood, MA, 2005.

[17] Laird Technologies Inc., Tgrease 2500 Series Thermal Grease, http://www.lairdtech.com/brandworld/library/THR-DS-Tgrease\%202500\%200710.pdf, 2010. 
[18] S. H. Taylor and G. S. V, "Level-Set Shape Reconstructio of Binary Permittivity Distributions using Near-Field Focusing Capacitance Measurements," Measurements Science and Technology, vol. 25, no. 10, p. 105602, 2014. 


\section{List of Figures}

Figure 1. Conceptual illustration of thermal test vehicle with embedded capacitive sensors.

Figure 2. Illustration of solution algorithm objective.

Figure 3. Prototype embedded sensor system manufactured on printed circuit boards.

Figure 4. Example calibration curves for a single capacitance sensor, with $J_{1}=0.0238, K_{1}=0.0957, K_{2}-$ $J_{2}=319$.

Figure 5. Experimental case with increasing load in the northwest and northeast locations. Grayscale range is from white at $\eta=2$ to black at $\eta=0$, with $H_{r e f}=100 \mu \mathrm{m}$.

Figure 6. Experimental case with voided grease layer: (a) Optical image of original voids in dispensed grease (red outline indicates top electrode); (b) void locations identified from optical image; (c) void fraction map obtained from the solution algorithm after assembly. Grayscale range is from white at $\chi=0$ to black at $\chi=0.5$, with $H_{r e f}=100 \mu \mathrm{m}$.

Figure 7. Experimental results for initially voided grease layer with increasingly non-uniform pressure (red outline indicates top electrode). 